\title{
Urinary neopterin and indices of renal involvement in rheumatoid arthritis
}

\author{
Manal A. M. Mandour*, Nadia M. Ismail**, Mohammed A. Sobh*** \\ Departments of Medical Biochemistry*, Rheumatology and \\ Rehabilitation**, and Internal Medicine*** \\ Faculty of Medicine, Assiut University
}

\begin{abstract}
There is increasing evidence that renal disease is presumed to be one of the main causes of mortality among rheumatoid arthritis (RA) patients. Thus, it is important to periodically carryout sensitive and reliable measures for renal function to pickup the earliest signs of renal dysfunction. In this regard, the aim of the present work is to look for the most predictable urinary index for early renal involvement in RA. Moreover, the study tried to find whether urinary neopterin levels give an indication about the renal affection in $R A$.

Urine samples were obtained from $49 R A$ patients. Most of the studied RA patients (91.84\%) were on combined therapy [non-steroidal anti-inflammatory drugs (NSAIDs), disease modifying anti-rheumatic drugs (DMARDs), and/or steroids]. The activity of the disease was evaluated on the basis of clinical and routine investigations. In addition, 15 urine samples were obtained from healthy controls. For all the studied groups traditional kidney function tests were performed. Specific urinary measures for renal function were determined namely, microalbuminuria, beta-2-microglobulin $\left(\beta_{2} M\right)$, $N$-acetyl glucosaminidase ( $\left.N A G\right)$, and gamma glutamyl transferase (GGT). Furthermore, urinary neopterin levels were estimated in all the studied groups. Receiver Operating Characteristics (ROC) curve was used to select the best cutoff values for identifying the sensitivity and the specificity of the diagnostic test.

The present study recorded 23 out of 49 (46.93\%) RA patients had low creatinine clearance (CrCl), 13 (26.53\%) had macroalbuminuria, 17 (34.69\%) had microalbuminuria, and $9(18.36 \%)$ had haematuria. A significant elevation of the levels of microalbumin, $\beta_{2} M$, and neopterin, and activities of $N A G$ and GGT was revealed in urine of RA patients as compared to control group. However, there were only an increase in the levels of microalbuminuria and urinary neopterin in $R A$ patients with extra-articular manifestations as compared to RA patients without. On the other hand, RA patients at risk of renal affection (with macroalbuminuria and/or low $\mathrm{CrCl}$ ) had high microalbuminuria and $\beta_{2} M$ levels. Also, the significant increase of the activities of $N A G$ and GGT was observed in those patients when compared to those with normoalbuminuria and/or normal $\mathrm{CrCl}$. Regarding the ROC plot, the area under the curve $(A U C)>0.7$ indicates a discriminating strength of statistical significance. ROC plot analysis showed that microalbuminuria, $\beta_{2} M, N A G$, GGT and
\end{abstract}


neopterin had discriminating power for renal affection among RA patients (AUC $0.90,0.956,0.77,0.92$, and 0.71 respectively). Urinary neopterin was positively correlated with ESR, urea, microalbumin, and urinary $\beta_{2} M(r=0.6, p<0.001 ; r=0.31$, $p<0.05 ; r=0.51, p<0.001$ and $r=0.43, p<0.01$ respectively).

In conclusion, the regular urine examination by simple and sensitive indices for early identification of renal affection in RA could guide the clinicians for a suitable line of therapy. The elevation of urinary neopterin levels was related to extraarticular manifestations as well as some kidney function tests. Such findings might clarify to some extent the role of urinary neopterin as an indicator of renal involvement in RA patients particularly those with active disease.

\section{INTRODUCTION}

Rheumatoid arthritis (RA) is a systemic disease, which mainly affects the joints. It can also lead to extra-articular organ manifestations. There is increasing evidence that renal disease is presumed to be one of the main causes of mortality among RA patients ${ }^{(1 \& 2)}$.

Clinically, manifest renal involvement in RA has been commonly attributed to secondary amyloidosis and renal vasculities ${ }^{(3 \& 4)}$. Nephrotoxic medication as nonsteroidal anti-inflammatory drugs (NSAIDs) some disease modifying anti-rheumatic drugs (DMARDs), and even anti-TNF-alpha therapy is the other major etiology of kidney involvement ${ }^{(5,6 \& 7)}$. The incidence of nephropathy in RA patients is not known, since RA patients frequently show subclinical renal dysfunction ${ }^{(3)}$.

Traditionally, the approach to detect any renal disease has always been first the urinary examination followed by histopathological examination. Although renal biopsy is the gold standard, it is being invasive and cannot be done at regular interval to diagnose renal disease early ${ }^{\left({ }^{(8)}\right.}$.
Many reports have looked at glomerular and tubular proteinuria as markers of nephropathy in RA. These were preferred over serum creatinine $(\mathrm{Cr})$ and $\mathrm{Cr}$ clearance $(\mathrm{CrCl})$, the usually available simple tests. The latter tests lack the sensitivity especially in RA patients due to their reduced muscle mass, whereas, proteinuria is more sensitive to detect renal diseases ${ }^{(9 \& 10)}$. Microalbuminuria is defined as a persistent values of albumin in urine between $>30-<300$ $\mathrm{mg} /$ day ${ }^{(11)}$. It is considered as a simple and sensitive test to detect early subclinical renal dysfunction as drug induced renal damage in RA (3\&12)

A protein of low molecular weight, beta-2-microglobulin $\left(\beta_{2} \mathrm{M}\right)$ is composed of 100 amino acids (13). Because of its small size, $\beta_{2} \mathrm{M}$ is filtered at the glomerulus and almost totally reabsorbed and catabolised by proximal tubular cells. Therefore, serum and urine $\beta_{2} \mathrm{M}$ levels were determined to assess glomerular and tubular damage respectively ${ }^{(14)}$. In addition, some enzyme activities as Nacetyl glucosaminidase (NAG, EC 3.2.1.30) and gamma glutamyl transferase (GGT, EC 2.3.2.2) were 
determined and used as early sensitive indicators for kidney damage in RA patients ${ }^{(15 \& 16)}$. They are proximal tubular enzymes whose urinary levels have been reported to increase during episodes of renal damage ${ }^{(17 \& 18)}$.

A pyrazino-pyrimidine compound, neopterin serves as a marker of cellular immune system activation. It is of clinical value compared to other laboratory findings to define prognosis, extra-articular manifestations and efficacy of ongoing therapy in RA (19\&20). Nevertheless, few reports documented the increased serum and urinary neopterin levels in different renal diseases ${ }^{(21,22 \& 23)}$

Irrespective of the causes of renal affection in RA, it is important to periodically carryout sensitive and reliable measures for renal function to pickup the earliest signs of renal dysfunction. In this regard, the present work aimed to investigate specific urinary indices besides the routine kidney function tests in RA. In addition, the statistical study looked for the most predictable urinary index for early renal involvement in RA. Moreover, the study tried to find whether urinary neopterin levels give an indication about the renal affection in RA.

\section{PATIENTS \& METHODS}

The current study included 64 subjects. Forty-nine patients with RA (32 female and 17 male) aged 25-65 years $($ mean $\pm \mathrm{SE}=44.64 \pm 1.43$ ) admitted to Rheumatology and Rehabilitation Department, Assiut
University Hospital. Also, 15 healthy volunteers matched by sex and ages were considered as a control group. All RA patients fulfilled the American Rheumatism Association, 1987 revised criteria (24). They were selected by the criteria of no casts and serum creatinine and blood urea in the normal range. Most of the studied RA patients $(91.84 \%)$ were on combined therapy (NSAIDs, DMARDs and/or steroids).

Exclusion criteria: Subjects with other autoimmune disease, gouty arthritis, past or present history of renal affection, essential hypertension, liver diseases, diabetes mellitus, malignancy and pregnancy were excluded.

Clinical investigations: Thorough medical history was taken. The signs of activity of the disease were detected by: 1- Morning stiffness (min.) according to Mallaya and Mace (25). 2- Joint tenderness was assessed by Ritchie articular index ${ }^{\mathbf{( 2 6 )}}$. The following joints were considered as a single joint, namely tempromandibular joints, acromioclavicular joints, metacarpophalangeal joints, proximal interphalangeal joints of each hand, and metatarsophalangeal joints of each foot. 3- Pain severity was evaluated according to American Collage of Rheumatology recommendation (27). 4- Functional capacity was performed ${ }^{(28)}$.

Routine

Investigations:

Radiological assessment was performed by plain X-ray ${ }^{(29)}$. Abdominal ultrasonography was assessed to exclude congenital abnormalities of kidneys, polycystic 
kidney disease, stones, hydronephrotic kidney, and tumors. Erythrocyte sedimentation rate (ESR), rheumatoid factor (RF), peripheral haemogram, and complete urinalysis were evaluated. Routine kidney function tests were performed namely, serum and urinary $\mathrm{Cr}$ (by Jaffe reaction), $\mathrm{CrCl}$ and macroalbuminuria. Determination of blood glucose levels, liver function tests, and ECG were done to exclude the presence of some diseases.

\section{Specific}

Biochemical

Investigations: Early morning urine samples were collected for measurement of specific urinary indices for renal function. Microalbuminuria levels were estimated by an indirect solid phase enzyme linked immunosorbent assay (ELISA) kit (30) (ORGENTEC Diagnosticka Gmbh, Mainz, Germany). Determination of $\beta_{2} \mathrm{M}$ levels according to Crisp et al. ${ }^{(31)}$ by ELISA kit (DRG Intrnational Inc, USA). NAG activities were measured enzymatically by using 4-nitrophenyl$N$-acetyl- $\beta$-D-glucosaminide as substrate (32). Glycyl-glycine and Lglutamyl-3-carboxy-p-nitroanilide were used as substrates for estimation of GGT activities (33) by kit (QUIMICA CLINICA APLICADA SA, Spain). Neopterin levels were determined using ELISA kit ${ }^{(34)}$ (IBL, Gmbh, Hamburg, Germany; RE59321).

Statistical analysis: Data were analyzed using Prism software program, graphPad version $3.0^{(35)}$. Data of clinical and routine laboratory parameters were expressed as mean \pm $\mathrm{SE}$ or number. Statistical differences were calculated with Man-Whittney test and Kruskal-Wallis test (Dunn's multiple comparison post-test) were used for non-parameteric values. Spearman's rank correlation coefficient was used for evaluating the correlation between the biochemical variables. $\mathrm{p}<0.05$ was considered a significant. The threshold value for optimal sensitivity and specificity of the specific urinary indices and neopterin was determined by Receiver Operating Characteristics (ROC) curve, which was plotted by calculating sensitivity and 1specificity at several cutoff points. The ROC curve can be used to select the best cutoff for identifying the sensitivity and the specificity of the diagnostic test. This is useful for comparing the ability of test to discriminate between the RA patients at risk of renal affection and the remaining RA patients. The area under the curve (AUC) of the ROC plots range from 1.0 (perfect separation of test values into two groups) to 0.5 (no distributional difference). An AUC $>0.7$ indicates a discriminating strength of statistical significance; an AUC $>0.8$ indicates excellent discriminating power of the test ${ }^{(36)}$.

\section{RESULTS}

Clinical characteristics of RA patients are shown in table (1). Table (2) demonstrates the routine investigations of the disease activity, and liver as well as kidney function tests. Considering that most RA patients $(91.84 \%)$ received combined therapy (NSAIDs, DMARDs and/or 
steroids), the present study recorded $23(46.93 \%)$ out of 49 RA patients had low $\mathrm{CrCl}, 13(26.53 \%)$ had macroalbuminuria, $17(34.69 \%)$ had microalbuminuria, and 9 (18.36\%) had haematuria.

A significant elevation of the levels of microalbumin, $\beta_{2} \mathrm{M}$, and neopterin, and activities of NAG and GGT was revealed in urine of RA patients as compared to control group (table 3). However, there were only increase in the levels of microalbuminuria and neopterin in RA patients with extra-articular manifestations as compared to RA patients without (table 4). On the other hand, table (5) clarified that RA patients with macroalbuminuria and/or low $\mathrm{CrCl}$ had higher microalbuminuria and $\beta_{2} \mathrm{M}$ levels. Also, a significant increase of the activities of NAG and GGT was observed in those pateints when compared to those with normoalbuminuria and/or normal $\mathrm{CrCl}$. The enzymes NAG and GGT could differentiate between RA patients with macroalbuminuria and those with microalbuminuria (table 6). From the ROC curves (figure 1), the best cut-off values were identified; and sensitivity, specificity, positive predictive value $(+\mathrm{PV})$, negative predictive value (-PV), to discriminate RA patients at risk of renal affection from the remaining RA patients were showed in table (7).

Table (1): Clinical characteristics of RA patients.

\begin{tabular}{|l|c|}
\hline Item & RA (n= 49) \\
\hline Duration of disease (year) & $6.17 \pm 0.89$ \\
\hline Treatment: & \\
Combined therapy (NSAIDs,DMARDs and/or Steroids) & $45(91.84 \%)$ \\
NSAIDs & $47(95.91 \%)$ \\
DMARDs & $36(73.46 \%)$ \\
Steroids & $36(73.46 \%)$ \\
\hline Morning stiffness (min.) & $88.8 \pm 6.3$ \\
\hline Articular index (AI) & $19.34 \pm 9.39$ \\
\hline Functional capacity: & \\
Grade I & $15(30.6 \%)$ \\
Grade II & $24(49.0 \%)$ \\
Grade III & $9(18.4 \%)$ \\
Grade IV & $1(2.0 \%)$ \\
\hline Extra-articular manifestations & $23(46.9 \%)$ \\
\hline
\end{tabular}

Data are expressed as mean \pm SE or number (percentage). NSAIDs, non-steroidal antiinflammatory drugs; DMARDs, disease modifying anti-rheumatic drugs. 
Table (8) demonstrates the correlation between the studied biochemical parameters and other clinical and laboratory investigations. Urinary neopterin was positively correlated with ESR, urea, microalbuminuria, and urinary $\beta_{2} \mathrm{M} \quad(\mathrm{r}=0.6, \mathrm{p}<0.001$; $\mathrm{r}=0.31, \mathrm{p}<0.05 ; \mathrm{r}=0.51, \mathrm{p}<0.001$ and $\mathrm{r}=0.43, \mathrm{p}<0.01$ respectively). Also, urinary GGT was positively correlated with urea, microalbuminuria, and urinary $\beta_{2} \mathrm{M}(\mathrm{r}=0.31, \mathrm{p}<0.05 ; \mathrm{r}=0.7$, $\mathrm{p}<0.001$ and $\mathrm{r}=0.6, \quad \mathrm{p}<0.001$ respectively). Urinary NAG was positively correlated with both microalbuminuria $(\mathrm{r}=0.48, \quad \mathrm{p}<0.01)$ and urinary $\beta_{2} \mathrm{M} \quad(\mathrm{r}=0.41, \mathrm{p}<0.01)$. Furthermore, there was positive correlation between urinary $\beta_{2} \mathrm{M}$ and microalbuminuria $(\mathrm{r}=0.58, \mathrm{p}<0.001)$.

Table (2): Routine investigations for RA patients.

\begin{tabular}{|c|c|}
\hline Item & RA $(n=49)$ \\
\hline \multicolumn{2}{|l|}{ X ray } \\
\hline Grade I & $5(10.2 \%)$ \\
\hline Grade II & $20(40.8 \%)$ \\
\hline Grade III & $17(34.7 \%)$ \\
\hline Grade IV & $7(14.3 \%)$ \\
\hline \multicolumn{2}{|l|}{ RF (IU) } \\
\hline$-v e$ & $18(36.7 \%)$ \\
\hline$+v e$ & $31(63.3 \%)$ \\
\hline ESR1 & $63.29 \pm 4.18$ \\
\hline $\mathrm{Hb}(\mathrm{g} / \mathrm{dl})$ & $10.95+0.22$ \\
\hline$W B C s\left(x 10^{3} / \mathrm{mm}^{3}\right)$ & $7.48 \pm 0.26$ \\
\hline Platelets $\left(\times 10^{3} / \mathrm{mm}^{3}\right)$ & $276.38 \pm 12.51$ \\
\hline Serum bilirubin $(\mu \mathrm{mol} / \mathrm{L})$ & $7.1+0.46$ \\
\hline AST (U/L) & $23.82 \pm 1.31$ \\
\hline $\operatorname{ALT}(\mathrm{U} / \mathrm{L})$ & $19.02 \pm 0.94$ \\
\hline Serum albumin $(\mathrm{g} / \mathrm{L})$ & $36.73+0.56$ \\
\hline Macroalbuminuria (g/L) & $0.27 \pm 0.06$ \\
\hline Urinary RBCs & $9(18.36 \%)$ \\
\hline Urinary pus & $4(8.16 \%)$ \\
\hline Serum urea $(\mathrm{mmol} / \mathrm{L})$ & $4.74 \pm 0.19$ \\
\hline Serum creatinine $(\mu \mathrm{mol} / \mathrm{L})$ & $60.08 \pm 2.07$ \\
\hline Creatinine clearance $(\mathrm{ml} / \mathrm{min})$. & $97.62+5.31$ \\
\hline
\end{tabular}

Data are expressed as mean $\pm \mathrm{SE}$ or number (percentage). RF, rheumatoid factor; ESR1, erythrocyte sedimentation rate at first hour; Hb, hemoglobin; WBCs, white blood cells; AST, aspartate transaminase; ALT, alanine transaminase; RBCs, red blood cells. 
Table (3): Specific urine parameters of control and RA groups.

\begin{tabular}{|c|c|c|c|c|}
\hline Parameters & Control $(n=15)$ & RA (n=49) & $\boldsymbol{U}$ & $\boldsymbol{P}$ \\
\hline $\begin{array}{l}\text { Microalb. }(\mu \mathrm{g} / \mathrm{ml}) \\
\text { Mean } \pm S E \\
\text { Median }\left(25^{\text {th }}, 75^{\text {th }} \text { percentile }\right)\end{array}$ & $\begin{array}{l}4.57 \pm 1.7 \\
1.5(0.85,6)\end{array}$ & $\begin{array}{l}104.2 \pm 19.91 \\
35(2.75,300)\end{array}$ & 144.5 & 0.0004 \\
\hline $\begin{array}{l}\beta_{2} \mathrm{M}(\mu \mathrm{g} / \mathrm{g} \mathrm{Cr}) \\
\text { Mean } \pm S E \\
\text { Median }\left(25^{\text {th }}, 75^{\text {th }} \text { percentile }\right)\end{array}$ & $\begin{array}{l}467.6 \pm 15.1 \\
449(423,518.5)\end{array}$ & $\begin{array}{l}791 \pm 56.09 \\
767(457,1033) \\
\end{array}$ & 179 & 0.003 \\
\hline $\begin{array}{l}\mathrm{NAG}(\mathrm{IU} / \mathrm{g} \mathrm{Cr}) \\
\text { Mean } \pm S E \\
\text { Median }\left(25^{\text {th }}, 75^{\text {th }} \text { percentile }\right)\end{array}$ & $\begin{array}{l}6.03 \pm 0.166 \\
6.1(5.7,6.6) \\
\end{array}$ & $\begin{array}{l}8.46 \pm 0.26 \\
7.8(7.13,9.25)\end{array}$ & 15 & $<0.001$ \\
\hline $\begin{array}{l}\mathrm{GGT}(\mathrm{U} / \mathrm{g} \mathrm{Cr}) \\
\text { Mean } \pm \text { SE } \\
\text { Median }\left(25^{\text {th }}, 75^{\text {th }} \text { percentile }\right)\end{array}$ & $\begin{array}{l}12.1 \pm 0.91 \\
13(8.5,15.3)\end{array}$ & $\begin{array}{l}28.11 \pm 1.66 \\
27(17.7,39)\end{array}$ & 42 & $<0.001$ \\
\hline $\begin{array}{l}\text { Neopterin }(\mu \mathrm{mol} / \mathrm{mol} \mathrm{Cr}) \\
\text { Mean } \pm S E \\
\text { Median }\left(25^{\text {th }}, 75^{\text {th }} \text { percentile }\right)\end{array}$ & $\begin{array}{l}162 \pm 16.01 \\
125(117.5,250)\end{array}$ & $\begin{array}{l}316.7 \pm 16.89 \\
300(250,372.5)\end{array}$ & 67.5 & $<0.01$ \\
\hline
\end{tabular}

Microalb., microalbuminuria; $\beta 2 \mathrm{M}, \quad \beta$-2-microglobulin; $\mathrm{NAG}, \quad \mathrm{N}$-acetyl glucosaminidase; GGT, gamma-glutamyl transpeptidase; U, indicates Mann-Whitney value. 
Table(4): Specific urine parameters in relation to extra-articular manifestations.

\begin{tabular}{|c|c|c|c|c|}
\hline \multirow[b]{2}{*}{ Parameters } & \multirow[b]{2}{*}{ Control $(n=15)$} & \multicolumn{2}{|c|}{ RA $(n=49)$} & \multirow[b]{2}{*}{$\mathbf{K}-\mathbf{W}$} \\
\hline & & $\begin{array}{c}\text { No extra-art. } \\
(n=27)\end{array}$ & $\begin{array}{c}\text { Extra-art. } \\
(\mathrm{n}=22)\end{array}$ & \\
\hline $\begin{array}{l}\text { Microalb. }(\mu \mathrm{g} / \mathrm{ml}) \\
\text { Mean } \pm S E \\
\text { Quartiles }\end{array}$ & $\begin{array}{l}4.57 \pm 1.7 \\
1.5(0.85,6)\end{array}$ & $\begin{array}{c}{ }^{*} \\
55.2 \pm 19.39 \\
20(1.0,52.5)\end{array}$ & $\begin{array}{r}{ }^{* * * \#} \\
164.3 \pm 33.69 \\
40(30,337.5)\end{array}$ & 19.53 \\
\hline $\begin{array}{l}\beta_{2} \mathrm{M}(\mu \mathrm{g} / \mathrm{g} \mathrm{Cr}) \\
\text { Mean } \pm S E \\
\text { Quartiles }\end{array}$ & $\begin{array}{l}467.6 \pm 15.1 \\
449(423,518.5)\end{array}$ & $\begin{array}{l}{ }^{*} \\
677.8 \pm 55.37 \\
802(442.5,963)\end{array}$ & $\begin{array}{l}{ }^{* * *} \\
929.9 \pm 98.5 \\
829(496,1241)\end{array}$ & 13.52 \\
\hline $\begin{array}{l}\text { NAG }(\mathrm{IU} / \mathrm{g} \mathrm{Cr}) \\
\text { Mean } \pm S E \\
\text { Quartiles }\end{array}$ & $\begin{array}{l}6.03 \pm 0.166 \\
6.1(5.7,6.6)\end{array}$ & $\begin{array}{l}{ }^{* * * *} \\
7.96+0.28 \\
7.4(7.02,9.2)\end{array}$ & $\begin{array}{l}{ }^{* * *} \\
9.08 \pm 0.45 \\
8.6(7.5,10.1)\end{array}$ & 34.95 \\
\hline $\begin{array}{l}\text { GGT }(\mathrm{U} / \mathrm{g} \mathrm{Cr}) \\
\text { Mean } \pm S E \\
\text { Quartiles }\end{array}$ & $\begin{array}{l}12.1 \pm 0.91 \\
13(8.5,15.3)\end{array}$ & $\begin{array}{l}26.3 \pm 1.95^{* * *} \\
25(17.7,35)\end{array}$ & $\begin{array}{l}\quad{ }^{* * *} \\
30.33 \pm 2.8 \\
29(17.4,42)\end{array}$ & 27.61 \\
\hline $\begin{array}{l}\text { Neopterin } \\
(\mu \mathrm{mol} / \mathrm{mol} \mathrm{Cr}) \\
\text { Mean } \pm S E \\
\text { Quartiles }\end{array}$ & $\begin{array}{l}162 \pm 16.01 \\
125(117.5,250)\end{array}$ & $\begin{array}{l}{ }^{* *} \\
277.1 \pm 20.44 \\
265 .(225,337)\end{array}$ & $\begin{array}{l}353 \pm 15^{* * * \#} \\
311.5(294.3,531)\end{array}$ & 27.9 \\
\hline
\end{tabular}

Microalb., microalbuminuria; $\beta 2 \mathrm{M}, \quad \beta$-2-microglobulin; $\quad \mathrm{NAG}, \quad \mathrm{N}$-acetyl glucosaminidase; GGT, gamma-glutamyl transpeptidase; Extra-art., extra-articular manifestations; Quartiles, Median $\left(25^{\text {th }}, 75^{\text {th }}\right.$ percentile); K-W, indicates KruskalWallis value; ${ }^{*} \mathrm{p}<0.05,{ }^{* *} \mathrm{p}<0.01,{ }^{* * *} \mathrm{p}<0.001$ vs. control group; ${ }^{\#} \mathrm{p}<0.05$ vs. no extraart. group. 
Table (5): Specific urine parameters in relation to macroalbuminuria and/or creatinine clearance.

\begin{tabular}{|c|c|c|c|c|}
\hline \multirow[b]{2}{*}{ Parameters } & \multirow[b]{2}{*}{ Control $(n=15)$} & \multicolumn{2}{|c|}{ RA (n=49) } & \multirow[b]{2}{*}{$\mathbf{K}-\mathbf{W}$} \\
\hline & & $\begin{array}{c}\text { Macroalb. and/or } \\
\text { low CrCl } \\
(n=29)\end{array}$ & $\begin{array}{c}\text { Normoalb. and } \\
\text { normal CrCl } \\
(n=20)\end{array}$ & \\
\hline $\begin{array}{l}\text { Microalb. }(\mu \mathrm{g} / \mathrm{ml}) \\
\text { Mean } \pm \text { SE } \\
\text { Quartiles }\end{array}$ & $\begin{array}{l}4.57 \pm 1.7 \\
1.5(0.85,6)\end{array}$ & \begin{tabular}{l}
\multicolumn{1}{c}{$* * *$} \\
$166.6 \pm 28.31$ \\
$70(32.5,322.5)$
\end{tabular} & $\begin{array}{l}84 \pm 26.04 \\
20 \pm(1.3,172.5)\end{array}$ & 26.78 \\
\hline $\begin{array}{l}\beta_{2} \mathrm{M}(\mu \mathrm{g} / \mathrm{g} \mathrm{Cr}) \\
\text { Mean } \pm \text { SE } \\
\text { Quartiles }\end{array}$ & $\begin{array}{l}467.6 \pm 15.1 \\
449(423,518.5)\end{array}$ & $\begin{array}{l}{ }^{* * *} \\
1008 \pm 67.18 \\
956(793.6,1185)\end{array}$ & $\begin{array}{l}c \\
476.8 \pm 32.37 \\
447.5(418,471)\end{array}$ & 42.02 \\
\hline $\begin{array}{l}\text { NAG (IU/g Cr) } \\
\text { Mean } \pm S E \\
\text { Quartiles }\end{array}$ & $\begin{array}{l}6.03 \pm 0.166 \\
6.1(5.7,6.6)\end{array}$ & $\begin{array}{l}{ }^{* * *} \\
9.20 \pm 0.37 \\
8.6(7.6,10.15)\end{array}$ & $\begin{array}{l}{ }^{c}{ }^{* * \# \#} \\
7.4 \pm 0.22 \\
7.13(6.8,7.65)\end{array}$ & 41.63 \\
\hline $\begin{array}{l}\text { GGT (U/g Cr) } \\
\text { Mean } \pm S E \\
\text { Quartiles }\end{array}$ & $\begin{array}{l}12.1 \pm 0.91 \\
13(8.5,15.3)\end{array}$ & $\begin{array}{l}34 *{ }^{* * *} \\
34.87 \pm 1.79 \\
35(28.35,42)\end{array}$ & $\begin{array}{c}\text { \#\#\# } \\
18.30 \pm 1.27 \\
17.40(15.6,21.8)\end{array}$ & 44.41 \\
\hline $\begin{array}{l}\text { Neopterin } \\
(\mu \mathrm{mol} / \mathrm{mol} \mathrm{Cr}) \\
\text { Mean } \pm S E \\
\text { Quartiles }\end{array}$ & $\begin{array}{l}162 \pm 16.01 \\
125(117.5,250)\end{array}$ & $\begin{array}{l}354.1 \pm 23.83 \\
300(262,500)\end{array}$ & $\begin{array}{l}{ }^{* *} \\
262 \pm 17.06 \\
262.5(225,312.5)\end{array}$ & 27.5 \\
\hline
\end{tabular}

Microalb., microalbuminuria; $\quad \beta 2 \mathrm{M}, \quad \beta$-2-microglobulin; $\quad \mathrm{NAG}, \quad \mathrm{N}$-acetyl glucosaminidase; GGT, gamma-glutamyl transpeptidase; Macroalbu., macroalbuminuria; Normoalb., normoalbuminuria. Low $\mathrm{CrCl}$; creatinine clearance < $90 \mathrm{ml} / \mathrm{min}$; Normal $\mathrm{CrCl}$; creatinine clearance $\geq 90 \mathrm{ml} / \mathrm{min}$.; Quartiles, Median $\left(25^{\text {th }}\right.$ , $75^{\text {th }}$ percentile); K-W, indicates Kruskal-Wallis value; ${ }^{* *} \mathrm{p}<0.01,{ }^{* * *} \mathrm{p}<0.001$ vs. control group; ${ }^{\# \#}$ p $0.01,{ }^{\# \#} \mathrm{p}<0.001$ vs. macroalbuminuria and/or low $\mathrm{CrCl}$ group. 
Table (6): Specific urine parameters in relation to microalbuminuria.

\begin{tabular}{|c|c|c|c|c|}
\hline \multirow[b]{2}{*}{ Parameters } & \multicolumn{3}{|c|}{ RA $(n=49)$} & \multirow[b]{2}{*}{ K-W } \\
\hline & $\begin{array}{c}\text { Normoalbuminuria } \\
(\mathbf{n}=\mathbf{1 9})\end{array}$ & $\begin{array}{c}\text { Microalbuminuria } \\
(\mathrm{n}=17)\end{array}$ & $\begin{array}{c}\text { Macrolbuminuria } \\
(n=13)\end{array}$ & \\
\hline $\begin{array}{l}\beta_{2} \mathrm{M}(\mu \mathrm{g} / \mathrm{g} \mathrm{Cr}) \\
\text { Mean }+S E \\
\text { Quartiles }\end{array}$ & $\begin{array}{l}547.1+45.0 \\
459(428,733)\end{array}$ & \begin{tabular}{l}
\multicolumn{1}{c}{${ }^{* *}$} \\
$884.1+77.48$ \\
$956(482,1139)$
\end{tabular} & $\begin{array}{l}1026+140.5 \\
856(629,1260)\end{array}$ & 43.7 \\
\hline $\begin{array}{l}\mathrm{NAG}(\mathrm{IU} / \mathrm{g} \mathrm{Cr}) \\
\text { Mean } \pm \text { SE } \\
\text { Quartiles }\end{array}$ & $\begin{array}{l}7.67 \pm 0.28 \\
7.3(6.97,8.4) \\
\end{array}$ & $\begin{array}{l}8.22 \pm 0.30 \\
8.2(7.35,8.9)\end{array}$ & $\begin{array}{l}\text { **\# } \\
9.95 \pm 0.68 \\
9.7(8.2,11.2)\end{array}$ & 25.8 \\
\hline $\begin{array}{l}\text { GGT (U/g Cr) } \\
\text { Mean } \pm S E \\
\text { Quartiles }\end{array}$ & $\begin{array}{l}19.94 \pm 1.1 \\
17.7(16.85,25.2)\end{array}$ & $\begin{array}{l}27.03 \pm 2.77 \\
27.7(18.25,35)\end{array}$ & $\begin{array}{r}r * * \# \\
41.46 \pm 1.5 \\
41(39.5,45)\end{array}$ & 17.1 \\
\hline $\begin{array}{l}\text { Neopterin } \\
(\mu \mathrm{mol} / \mathrm{mol} \mathrm{Cr}) \\
\text { Mean } \pm S E \\
\text { Quartiles }\end{array}$ & $\begin{array}{l}240.8 \pm 14.59 \\
250(200,287.5)\end{array}$ & $\begin{array}{c}{ }^{* *} \\
357.1 \pm 23.25 \\
325(275,425)\end{array}$ & $\begin{array}{l}{ }^{* *} \\
375 \pm 41.60 \\
375(250,537.5)\end{array}$ & 14.3 \\
\hline
\end{tabular}

$\beta 2 \mathrm{M}, \beta$-2-microglobulin; NAG, N-acetyl glucosaminidase; GGT, gamma-glutamyl transpeptidase Quartiles, Median $\left(25^{\text {th }}, 75^{\text {th }}\right.$ percentile); K-W, indicates KruskalWallis value; ${ }^{* *} \mathrm{p}<0.01,{ }^{* * *} \mathrm{p}<0.001$ vs. normoalbuminuria; ${ }^{\#} \mathrm{p}<0.05$ vs. microalbuminuria. 


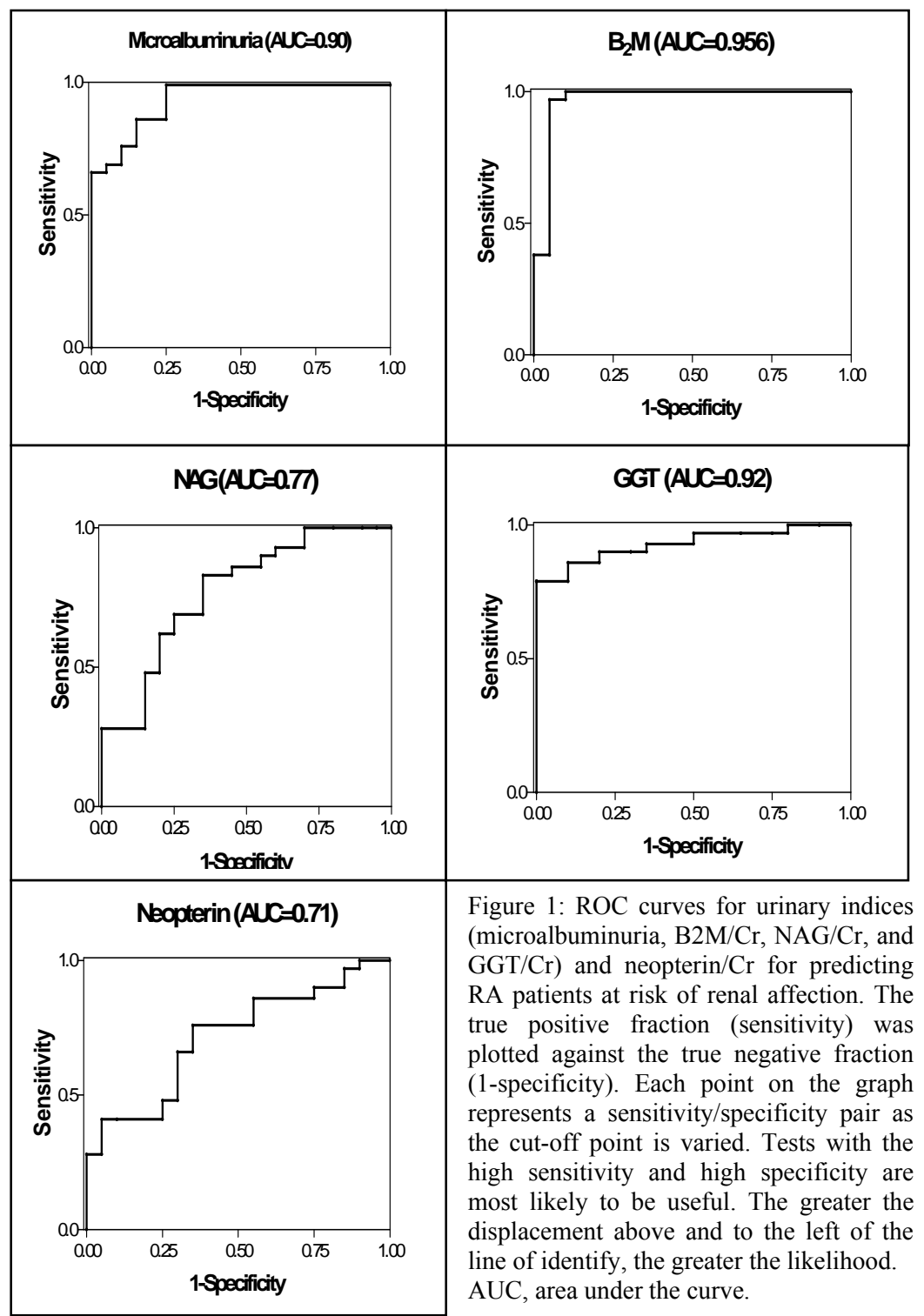


Table (7): Sensitivity, specificity, positive predictive value (+PV), negative predictive value (-PV), according to the best cut-off value of urinary indices to discriminate RA patients at risk of renal affection from the remaining $R A$ patients.

\begin{tabular}{|l|l|c|c|c|l|}
\hline Parameter & \multicolumn{1}{|c|}{ Cut-off value } & Sensitivity\% & $\begin{array}{c}\text { Specificity } \\
\mathbf{\%}\end{array}$ & $\begin{array}{c}+\mathbf{P V} \\
\mathbf{\%}\end{array}$ & $\begin{array}{c}\text {-PV } \\
\mathbf{\%}\end{array}$ \\
\hline Microalb. & $30 \mu \mathrm{g} / \mathrm{ml}$ & 75.86 & 85 & 88 & 70.8 \\
\hline$\beta_{2} \mathrm{M}$ & $518 \mu \mathrm{g} / \mathrm{g} \mathrm{Cr}$ & 96.55 & 95 & 96.6 & 95 \\
\hline $\mathrm{NAG}$ & $7.4 \mathrm{IU} / \mathrm{g} \mathrm{Cr}$ & 75.87 & 60 & 73.3 & 63.2 \\
\hline $\mathrm{GGT}$ & $23 \mathrm{U} / \mathrm{g} \mathrm{Cr}$ & 86.21 & 80 & 86.2 & 80 \\
\hline Neopterin & $275 \mu \mathrm{mol} / \mathrm{mol} \mathrm{Cr}$ & 65.52 & 65 & 74.1 & 59.1 \\
\hline
\end{tabular}

Microalb., microalbuminuria; $\beta 2 \mathrm{M}, \beta$-2-microglobulin; NAG, N-acetyl glucosaminidase; GGT, gamma-glutamyl transpeptidase.

Table (8): Correlations between the studied parameters among RA patients.

\begin{tabular}{|l|l|l|l|l|l|}
\hline Item & Microalb. & $\boldsymbol{\beta}_{\mathbf{2}} \mathbf{M}$ & NAG & GGT & Neopt. \\
\hline Age & -0.15 & 0.03 & -0.003 & -0.21 & 0.11 \\
\hline Disease duration & 0.033 & 0.22 & $0.33^{*}$ & 0.113 & 0.02 \\
\hline Morning stiffness & 0.025 & 0.014 & 0.145 & -0.14 & 0.10 \\
\hline$A I$ & 0.014 & 0.07 & 0.135 & 0.031 & 0.19 \\
\hline$E S R$ & 0.17 & 0.092 & 0.07 & 0.067 & $0.60^{* *}$ \\
\hline$R F$ & -0.27 & -0.065 & -0.19 & 0.096 & -0.079 \\
\hline$H b$ & -0.014 & 0.07 & 0.23 & 0.21 & -0.02 \\
\hline Serum urea & 0.22 & 0.16 & 0.07 & $0.31^{*}$ & $0.38^{* *}$ \\
\hline Serum creatinine & 0.21 & 0.28 & 0.26 & 0.08 & 0.03 \\
\hline Microalb. & & $0.58^{* * *}$ & $0.48^{* *}$ & $0.70^{* * *}$ & $0.51^{* * *}$ \\
\hline$\beta_{2} \mathrm{M}$ & & & $0.41^{* *}$ & $0.61^{* * *}$ & $0.43^{* *}$ \\
\hline$N A G$ & & & & 0.37 & 0.26 \\
\hline$G G T$ & & & & & 0.25 \\
\hline
\end{tabular}

Microalb., microalbuminuria; $\beta 2 \mathrm{M}, \quad \beta$-2-microglobulin; $\mathrm{NAG}, \quad \mathrm{N}$-acetyl glucosaminidase; GGT, gamma-glutamyl transpeptidase; AI, articular index; ESR, erythrocyte sedimentation rate; $\mathrm{RF}$, rheumatoid factor; $\mathrm{Hb}$, hemoglobin. 


\section{DISCUSSION}

The present investigation determined urinary excretion of $\beta_{2} \mathrm{M}$, NAG, and GGT as markers of tubular damage. Macroalbuminuria and microalbuminuria were assayed as indicators of glomerular dysfunction. Serum $\mathrm{Cr}, \mathrm{CrCl}$, and blood urea were determined to assess glomerular filtration. In the present study 23 out of $49(46.93 \%)$ RA patients had low $\mathrm{CrCl}, \quad 13 \quad(26.53 \%) \quad$ had macroalbuminuria, 17 (34.69\%) had microalbuminuria, and 9 (18.36\%) had haematuria; considering that most of the studied RA patients (91.84\%) were on combined therapy (NSAIDs, DMARDs and/or steroids).

Niederstädt et al. ${ }^{(12)}$ reported that $32 \%$ of RA patients had pathological albuminuria. Pederson et al. ${ }^{(37)}$ and Nordin et al. ${ }^{(38)}$ recorded $27.7 \%$ of RA patients with microalbuminuria. Glomerular and tubular proteinuria as markers of nephropathy were preferred over serum $\mathrm{Cr}$ and $\mathrm{CrCl}$ (9\&39), which have been shown to lack sensitivity to detect renal dysfunction in RA. This is attributed to loss of muscle mass and hence $\mathrm{Cr}$ production as also to altered renal handling of $\mathrm{Cr}$ due to either intrinsic renal disease or drug therapy ${ }^{(\mathbf{1 2})}$.

The present findings revealed an elevation of microalbuminuria levels in all RA patients as compared to control group. The increase in the levels of microalbuminuria was detected in RA patients with extraarticular manifestations as compared to RA patients without. On the other hand, RA patients with macroalbuminuria and/or low $\mathrm{CrCl}$ had higher microalbuminuria levels than those with normoalbuminuria and/or normal $\mathrm{CrCl}$. The statistical analysis showed that microalbuminuria determination might be useful in predicting renal affection among RA patients (AUC $=0.90, \quad+\mathrm{PV}=88 \%$ and $\mathrm{PV}=70.8 \%$ ). These data are compatible with other previous studies (12,37\&38), which demonstrated that microalbuminuria is a simple and sensitive test to detect early subclinical renal dysfunction as during induced renal damage in RA (3). Microalbuminuria is defined as a persistent value of albumin in urine between $30-300 \mathrm{mg} /$ day ${ }^{(11)}$. It is considered a subclinical increase of the albumin excretion in urine, since values above $300 \mathrm{mg} /$ day are considered to represent overt proteinuria ${ }^{(40)}$. The present data did not observe any correlation between microalbuminuria levels and disease duration or disease activity among RA patients. However, other investigations suggested that the urinary excretion of albumin might reflect a systemic reaction in the acute

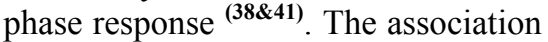
of albuminuria and disease duration has been reported and explained by two ways; either severe and longstanding RA tends to affect the kidneys and the systemic vascular permeability or patients with more severe and longstanding disease receive more nephrotoxic treatment (38)

Increased urinary excretion of $\beta_{2} \mathrm{M}$ reflects primarily tubular cell damage, 
although it has the disadvantage of instability in urine of $\mathrm{pH}<6.0^{(\mathbf{4 2 \& 4 3})}$. In the present investigation, RA patients showed significant increase in urinary $\beta_{2} \mathrm{M}$ levels than normal levels. Further increment of these levels was revealed among the RA patients at risk of renal affection, who had macroalbuminuria and/or low creatinine clearance. The ROC plot confirmed the role of $\beta_{2} \mathrm{M}$ as a sensitive predictor index for renal affection (AUC $=0.956$, sensitivity $=96.55 \%$, specificity $=95 \%$, $+\mathrm{PV}=96.6 \%$, and $-\mathrm{PV}=95 \%$ ). In addition, the positive correlation observed between microalbuminuria and excretion of $\beta_{2} \mathrm{M}$ might clarify the association of elevated urinary $\beta_{2} \mathrm{M}$ levels with renal involvement. Lewandowshki et al. ${ }^{(44)}$ reported that the concentration of urinary $\beta_{2} \mathrm{M}$ in RA patients was higher than in controls. Serum and urine $\beta_{2} \mathrm{M}$ levels were determined to assess glomerular and tubular damage respectively ${ }^{(14)}$, because $\beta_{2} \mathrm{M}$ is filtered at the glomerulus and almost totally reabsorbed and catabolised by proximal tubular cells ${ }^{(\mathbf{4 5})}$.

Recently, Pathan and Joshi (3) suggested that tubulointerstitial damage in RA was reflected by an increased excretion of $\beta_{2} \mathrm{M}$ and NAG and defective urinary concentrating capacity. The present study confirmed this suggestion, as it was found that urinary NAG activities were positively correlated with both microalbuminuria and urinary $\beta_{2} \mathrm{M}$ levels among RA patients. Moreover, the RA patients with macroalbuminuria and/or low $\mathrm{CrCl}$ showed higher urinary NAG activities than those patients without. The urinary NAG activities were significantly elevated in patients with macroalbuminuria as compared to those with microalbuminuria. The study revealed a positive correlation between urinary NAG activities and duration of disease. Iqbal et al. (46) observed a correlation between the total activity of urinary NAG and severity of the disease. The author evaluated isoenzymes of NAG (NAG$B$ and NAG-A) in urine and showed that the ratio of NAG-B to NAG-A significantly increases in severe cases of seropositive RA. The present results from ROC plot showed that urinary NAG activities had the lowest discriminating power of renal affection in RA patients (AUC $=0.77$, sensitivity $=75.87 \%$, specificity $=60 \%$, $+\mathrm{PV}=73.3 \%$, and $-\mathrm{PV}=63.2 \%$ ) than $\beta_{2} \mathrm{M}, \mathrm{GGT}$, and microalbuminuria. Electrophoresis divides urinary NAG into three isozymes, preA, $\mathrm{A}$, and $\mathrm{B}$ forms. The measurement of urinary NAG isozymes is useful to determine the type and severity of renal disease (47). Concerning RA, Iqbal et al. (46) suggested that subclinical renal injury without compromising renal function is a possibility in RA especially in severe cases of this disease. Also, they reported that it is unclear which factors lead to increased excretion of NAG (especially the isoenzyme form B) in RA patients.

GGT is a membrane-bound enzyme that is primarily synthesized in the brush border membrane of proximal tubules, and it is present in cell membranes of kidney, liver, and

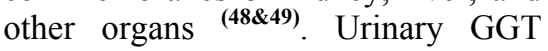
activity was used as an indicator of tubular damage for early renal 
injury $^{\mathbf{5 0})}$. In the current investigation the activities of urinary GGT were significantly increased more than two folds in RA patients than control group. The RA patients with macroalbuminuria and/or low $\mathrm{CrCl}$ had higher urinary activities than those without. During tubular injury, brush-border surface glycoproteins, which contain various enzymes, are shed and released into the urine ${ }^{(51)}$. Herein, for distinguishing the RA patients at high resk of renal affection from the remaining RA patients, the ROC curve detected an excellent discriminating power of urinary GGT $(\mathrm{AUC}=0.92, \quad$ sensitivity $=86.21 \%$, specificity $=80 \%, \quad+\mathrm{PV}=86.2$ and $\mathrm{PV}=80 \%$ ). A positive correlation between urinary GGT and both microalbuminuria and urinary $\beta_{2} \mathrm{M}$ levels was found. Rambabu et al. (15) observed a significant correlation between urinary GGT and serum albumin. They suggested that enhanced excretion of urinary GGT may be stimulated by decreased albumin concentration or oncotic pressure but does not appear to be due to leakage from plasma. Increased excretion of that protein implies injury to the brush border membrane with loss of microvillus structure. Losses of significant fraction of the microvillus surface area are also leads to reduced reabsorption and increased excretion of filtered proteins such as $\beta_{2} \mathrm{M}$ and retinal-binding protein ${ }^{(52)}$. Urinary GGT activities were positively correlated with serum urea in the present study. Hofmeister et al. (53) reported that the increase in enzymuria (as GGT) was associated with a drastic increase in serum urea nitrogen and $\mathrm{Cr}$. The authors concluded that the determination of only some urinary enzymes is sufficient for diagnosis of proximal tubule damage. On the other hand, highly reactive oxygen species play an important role in the etiology of tissue injury in RA ${ }^{(54)}$. Recently, GGT had been reported to be a marker of inflammation and oxidative stress $^{(\mathbf{5 5 \& 5 6})}$. During oxidative stress, GGT gene expression is increased,

The urinary levels of neopterin in RA were above the normal levels in the present study. The higher urinary levels were shown in RA patients with extra-articular manifestations than the patients without. Moreover, the data revealed that urinary levels of neopterin are positively correlated with ESR. All the aforementioned findings confirmed the strong relation between the excreted neopterin and the activity of RA as reported before $^{(\mathbf{5 7 , 5 8 \& 5 9 )}}$. Neopterin is of clinical value to define prognosis, extraarticular manifestations and efficacy of ongoing therapy (19\&20). The increased amounts of neopterin are produced by human monocytes/macrophages upon stimulation with the cytokine interferon $\gamma$. It is furthermore produced constitutively. The amount of neopterin secreted correlates with the capacity of the same cells to produce reactive oxygen species (60\&61). Therefore, measurement of neopterin concentration in the body fluids provides information about activation of T-helper cell 1 derived cellular immune activation ${ }^{(61)}$.

Neopterin is excreted in an unchanged form via kidneys ${ }^{(23)}$. In a 
study on chronic renal disease Godai et al. (21) suggested that the neopterin levels in serum are closely linked with glomerular filteration rate and yet the urinary neopterin/Cr ratios may reflect the predominance of increased tubular secretion of neopterin over the renal injury per se. Increasing serum neopterin concentrations and urinary neopterin/Cr ratios may reflect disordered cell-mediated immunity in the nephritic syndrome, irrespective of glomerular histology or interstitial cell infiltration ${ }^{\mathbf{( 2 2}}$. In the present work, no significant difference was detected regarding urinary levels of neopterin between RA patients with macroalbuminuria and/or low $\mathrm{CrCl}$ and those without. The statistical analysis from ROC curve to discriminate RA patients at risk of renal affection from the remaining $\mathrm{RA}$ patients showed that $\mathrm{AUC}=0.71$, sensitivity $=65.52$, specificity $=65$, and $+\mathrm{PV}=74.1$. A positive correlation between urinary levels of neopterin and both microalbuminuria levels and urinary $\beta_{2} \mathrm{M}$ levels among RA patients was obsereved. The present investigation did not identify the pathological etiology of renal affection in RA patients involved in the study. Thus, the present work suggested that the elevation of urinary neopterin in RA may be related to renal affection due to severity of the autoimmune disease itself or due to vasculities which is usually accompanied by perturbation of cellmediated immunity. All the previous reports lend further support to the notion that subclinical damage to kidney may occur in RA.
In conclusion, the regular urine examination by simple and sensitive indices for early identification of renal affection in RA could guide the clinicians for a suitable line of therapy. The elevation of urinary neopterin levels was related to extraarticular manifestations as well as some kidney function tests. This may clarify to some extent the role of urinary neopterin as an indicator of renal involvement in RA patients particularly those with active disease.

\section{REFERENCES}

1. Myllykangas-Luosujärvi RA, Aho K and Isomäki HA (1995):

Mortality in rheumatoid arthritis. Semin Arthritis Rheum., 25:193202.

2. Sihvonen S, Korpela M, Mustonen J, Laippala $P$ and Pasternack A (2004): Renal disease as a predictor of increased mortality among patients with rheumatoid arthritis. Nephron Clin. Pract., 96(4):c 105-6

3. Pathan E and Joshi VR (2004): Rheumatoid arthritis and the kidney. J. Assoc. Physicians India., 52: 488-94.

4. Schwarting $A$ and MarkerHermann E (2005): Renal manifestations of rheumatic diseases. Z. Rheumatol., 64(1): 18-25.

5. Pham $K$ and Hirschberg $R$ (2005): Global safety of coxibs and NSAIDs. Curr. Top. Med. Chem., 5(5): 465- 73.

6. Stokes MB, Foster K, Markowitz GS, Ebrahimi F, 
Hines W, Kaufman D, Moore B, Wolde D and D'Agati VD: Development of glomerulonephritis during antiTNF-alpha therapy for rheumatoid arthritis. Nephrol. Dial. Transplant., 20(7): 1400-6.

7. Sugii S 2005: Adverse effects of DMARDs in treatment of rheumatoid arthritis. Nippon Rinsho., 63 (Suppl. 1):566-72.

8. Nadkar MY and Londhey VA (2004): Investigating kidney involvement in rheumatoid arthritis. J. Assoc. Physicians India., 52: 447-8

9. Boers M, Dijkmans BAC, Breedveld FC and Mattie $H$ (1988): Errors in prediction of creatinine clearance in patients with rheumatoid arthritis. Br. J. Rheum., 27:233-35.

10. Salli L, Scalici G, Corrao S, Curiale $B$ and Salerno $L$ (1990): Microproteinuria as an index of initial renal lesion in patients with rheumatoid arthritis. Clin. Exp. Rheumatol. 8:397-400.

11. Bakris GL (1996): Microalbuminuria: prognostic implications. Curr. Opin. Nephrol. Hypertens. 5(3): 21923.

12. Niederstädt $C$, Happ E, Tatsis E, Schnabel $A$ and Steinhoff $J$ (1999): Glomerular and tubular proteinuria as markers of nephropathy in rheumatoid arthritis. Rheumatology 38:2833.

13. Berggard B, Bjork L, Cigen R, Logdberg $L$ (1980): $\beta_{2^{-}}$ Microglobulin. Scand. J. Clin. Lab. Invest. (Suppl.) 154: 13-25.
14. Mortada WL, Sobh MA, ElDefrawy MM and Farahat SE (2002): Mercury in dental restoration: is there a risk of nephrotoxicity? J. Nephrol., 15(2): 171-6.

15. Rambabu $K$, Alaiat $S M$ and Ziu MM (1988): Studies on urinary gamma-glutamyl transpeptidase in nephritic syndrome patients. Biochem. Med. Metab. Biol., 40(1):68-75.

16. Wiland $P$ and Szechinski $J$ (1999): N-acetyl-beta-Dglucosaminidase urinary excretion as an early indicator of kidney damage in rheumatoid arthritis patients starting on parenteral gold and DepoMedrone/placebo injections. Clin. Rheumatol., 18:106-13.

17. Sesso R, Neto JTM, Ferraz $M B$, Pereira AB and Ajzen $H$ (1991): Frequent abnormalities of renal function in patients with rheumatoid arthritis. Nephron 59:677-8.

18. Peng SZ, Zhang CS, Hu Y, Zhang J, Wei $M$, Liu $L$ and Wang Y (2004): Monitoring indexes for early renal injury in the workers exposed to mercury. Zhonghua Lao Dong Wei Sheng Zhi Ye Bing Za Zhi 22(2): 122-4.

19. Nassonov EL, Samsonov MIu, Chichasova NV, Imametdinova GR, Nikiforova EL, Aleksandrova EN, IlichStoyanovich O, Fux D and Vaxter H (1998): Neopterin: laboratory marker of cellular immunity activation in rheumatoid arthritis. Ter. Ark., 70 (5):28-31. 
20. Nassonov EL, Samsonov MIu, Tilz $G$ and Fuchs D (2000): Neopterin: new immunological marker of autoimmune rheumatic disease. Klin. Med. (Mosk.) 78(8): 43-6.

21. Godai $K$, Uemasu $J$ and Kawasaki H (1991): Clinical significance of serum and urinary neopterin in patients with chronic renal disease. Clin. Nephrol., 36(3): 141-6.

22. Oda $K$, Arai $T$ and Nagase $M$ (1999): Increased serum and urinary neopterin in nephritic syndrome indicate cell-mediated immune dysfunction. Am. J. Kidney Dis., 34(4): 611-7.

23. Berdowska $A$ and ZwirskaKorczala K (2001): Neopterin measurement in clinical diagnosis. J. Clin. Pharm. Ther.,26(5):319-29.

24. Arnett FC, Edworth SM, Bloch DA et al., (1988): The American Rheumatism Association 1987 revised criteria for the classification of rheumatoid arthritis. Arthritis Rheum., 31:315-24

25. Mallaya RK and Mace BEW (1981): The assessment of disease activity in rheumatoid arthritis using multivariate analysis. Rheumatol. Rehabilitation, 20(1): $14-17$.

26. Ritchie DM (1968): Clinical studies with an articular index for the assessment of joint tendeness in patients with rheumatoid arthritis. Q J Med New Series XXXVII, 147(37): 393 - 404.

27. Felson DT, Andason JJ, Bores M, Bombardia C, Frist D,
Goldsmith C, Mkatz L, Lightfoot RJR, Paulus $H$, Strand VE, Tugwell $P$, Weinblatt M, Williams ALJ, Wolfe F and Kiesza KS (1995): American college of rheumatology preliminary definition of improvement in rheumatoid arthritis. Arthritis Reum., 38: 727 - 35.

28. Hochberg MC, Chang RW, Dwosh I, Lindsey S, Pincus T and Wolfe F (1992): The American College of Rheumatology 1991 revised criteria for the classification of global functional status in rheumatoid arthritis. Arthritis Rheum.,35(5):498-502.

29. Scott DL, Houssein DA and Lasonen I (1995): Proposed modification to larsen's scoring methods for hand and wrist radiographs. Br. J. Rheumatol., 34: 56.

30. Mogensen CE (1984): Microalbuminuria predicts clinical proteinuria and early mortality in maturity-onset diabetes. New England journal of medicine 310: 356-60.

31. Crisp AJ, Coughlan RJ, Mackintosh D, Clark $B$ and Panayl GS (1983): $\beta_{2^{-}}$ Microglobulin plasma levels reflect disease activity in rheumatoid arthritis. J. Rheumtol., 10: 954-56.

32. Maruhn D (1976): Rapid colorimetric assay of $\beta$ galactosidase and $\mathrm{N}$-acetyl- $\beta$ Glucosaminidase in human urine. Clin. Chim. Acta 73: 453-61. 
33. Szasz G (1969): A kinetic photometric method for serum gamma-glutamyl transpeptidase. Clin. Chem., 15(2):124-36.

34. Westermann J, Thiemann F, Gerstner L, Tatzber F, Kozak I, Bertsch $T$ and Kruger $C$ (2000): Evaluation of a new simple and rapid enzyme-linked immunosorbent assay kit for neopterin determination. Clin. Chem. Lab. Med., 38(4): 345-53.

35. Motulsky HJ (1999): Analyzing data with graphPad prism, graph pad software Inc, San Diago CA, pp 207- 21.

36. Hanley JA and McNeil BJ (1982): The meaning and the use of the area under a receiver operating characteristic (ROC) curve. Radiology 143: 29-36.

37. Pederson LM, Nordin $H$, Svensson $B H$ and Bliddal $H$ (1995): Microalbuminuria in patients with rheumatoid arthritis. Ann. Rheum. Dis., 54: 189-92.

38. Nordin H, Pederson LM, Svensson $B H$ and Bliddal $H$ (1996): Microalbuminuria in rheumatoid arthritis. Ugeskr Laeger 158: 3141-3.

39. Nived O, Strutfelt G, Westling $H$ and White $T$ (1983): Is serum creatinine concentration a reliable index of renal function in rheumatic disease? Br. Med. J., 286: 684-5.

40. Jensen JS, Clausen P, BorchJohnsen $K$, Jensen $G$ and Feldt-Rasmussen B (1997): Detecting microalbuminuria by urinary albumin/creatinine concentration ratio. Nephrol.
Dial. Transplant., 12; Suppl. (2):6-9.

41. Shearman $C P$ and Gosling $P$ (1988): Microalbuminuria and vascular permeability. Lancet 2(8616):906-7.

42. Hordon LD, Bird HA and Cooper EH (1991): Renal tubular dysfunction in rheumatic diseases. Br. J. Rheumatol., 30: 115-18

43. Gella F-J and Capdevila M-J (1996): Determination of $\beta_{2^{-}}$ microglobulin in human serum and urine by latex turbidimetry. Pure\&Appl. Chem., 68: 1881-5.

44. Lewandowski B, Bernaka $K$, Kucharewicz B, Szimitkowski $M$ and Klimiuk PA (1996): Assessment of beta-2microglobulin concentration in serum and urine in rheumatoid arthritis. Rocz. Akad. Med. Bialymst., 41:482-91.

45. Morgan DB (1982): Assessment of renal tubular function and damage and their clinical significance. Ann.Clin. Biochem., 19:307-13.

46. Iqbal MP, Ali MM, Waqar MA and Mehboobali N (1998): Urinary N-acetyl-Dglucoseaminidase in rheumatoid arthriris. Exp. Mol. Med., 30: 165-9.

47. Furuhata $N$, Shiba $K$ and Nara N (1995): N-acetyl-beta-Dglucosaminidase. Nippon Rinsho 53(5): 1267-76.

48. Curto KA, Sweeny WE, Avner ED, Piesco NP and Curthoys NP (1988): Immunocytochemical localization of gammaglutamyltranspeptidase during 
fetal development of mouse kidney. J. Histochem. Cytochem., 36(2): 159-66.

49. Dominici S, Paolicchi A, Corti A, Maellaro E and Pompella A (2005): Prooxidant reactions promoted by soluble and cellbound gammaglutamyltransferase activity. Methods Enzymol., 401: 484501.

50. Westhuyzen J, Endre $Z \mathbf{H}$, Reece G, Reith DM, Saltissi D and Morgan TJ (2003): Measurement of tubular enzymuria facilitates early detection of acute renal impairment in the intensive care unit. Nephrol. Dial. Transplant 18: 543-55.

51. Ishii $\mathbf{N}$, Dgawa $Z$,and Itoh $H$ (1994): Diagnostic significance of urinary enzymes for diabetes mellitus and hypertension. Enzyme Protein 48: 174-82.

52. Guder WG and Hofman W (1992): Markers for the diagnosis and monitoring of renal tubules lesions. C. Nephrol., 38(Suppl.1): S3-S7.

53. Hofmeister $R$, Bhargava AS and Gunzel $P$ (1986): Value of enzyme determinations in urine for the diagnosis of nephrotoxicity in rats. Clin Chim Acta. 1986 Oct 31;160(2):163-7.

54. Mapp PI, Grootveld MC and Blake DR (1995): Hypoxia, oxidative stress and rheumatoid arthritis. Br. Med. Bull., 5:41936.

55. Yamada J, Tomiyama $H$, Yambe M, Koji Y, Motobe K, Shiina $K$ and Yamamoto $A$
(2006): Elevated serum levels of alanine amidotransferase and gamma glutamyltransferase are markers of inflammation and oxidative stress independent of the metabolic syndrome. Atherosclerosis 4 (under press)

56. Yue M, Ni Q, Yu CH, Ren KM, Chen WX and Li YM (2006):

Transient elevation of hepatic enzymes in volunteers after intake of alcohol. Hepatobiliary Pancreat. Dis. Int., 5(1): 52-5.

57. Kulich W (1993): Correlation of interleukin-2 receptor and neopterin excretion in rheumatoid arthritis. Clin. Rheumatol., 12(3): 387-91.

58. Mazzetti I, Grigolo B, Borz RM (1996): Serum copper/zinc superoxide dismutase levels in pateints with rheumatoid arthritis. Int. J. Clin. Lab. Res., 26(4):24549.

59. Altindag $Z$, Sahin $G$, Nanici I and Haoclikez F (1998): Urinary neopterin excretion and dihydropteridine reductase activity in rheumatoid arthritis. Rheumatol. Int., 18(3): 107-10.

60. Abou Deya SH, El-Lakany SA and Sharaki OA (1998): Advanced oxidation protein products, serum interleukin- 6 and urinary neopterin as markers of cell mediated immunity in relation to proteinuria in diabetic nephropathy. Clin. Chem. 6 (Suppl. 6), A8.

61. Murr C, Widner B, Wirleitner and Fuchs D (2002): Neopterin as a marker for immune system activation. Current Drug Metabolism 3: 175-187. 


\section{النيوترين البولى و مؤشرات تأثر الكلى فى مرضى الروماتويد المفصلى}

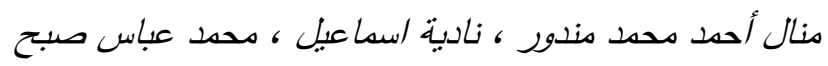

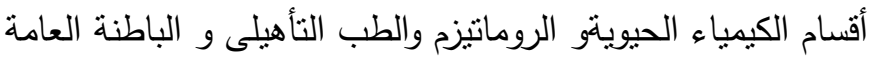

تعد أمراض الكلى واحدة من أسباب الوفاة لمرضى الروماتويد المفصلى. لذا فمن المهم أن نقوم دوريًا

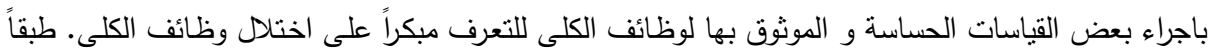
لذلك فان الدراسة الحالية بحثت عن أكثر المؤشرات توقعاً لتأثر الكلى المبكر فى الروماتويد المفصلى. بالاضافة

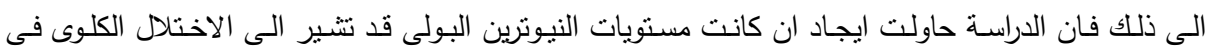

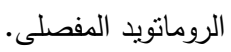

فى هذه الدراسـة تم الحصول على عينات بول من 9 ؛ مريضـاً بالروماتويد المفصلى وقد كان معظم

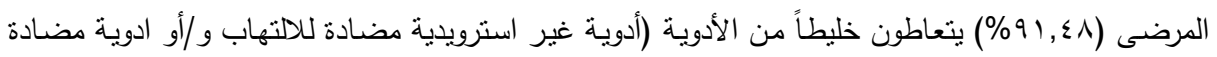

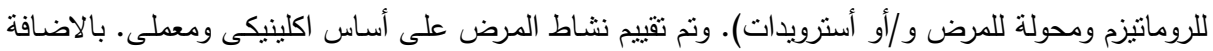

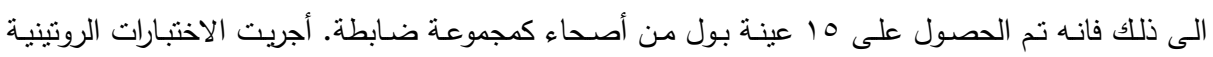

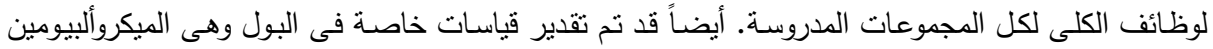

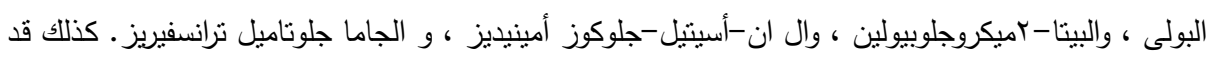

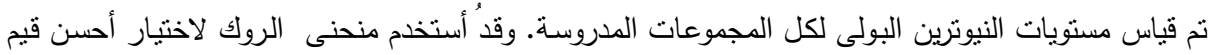

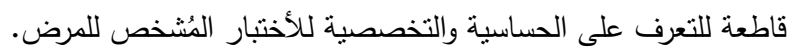

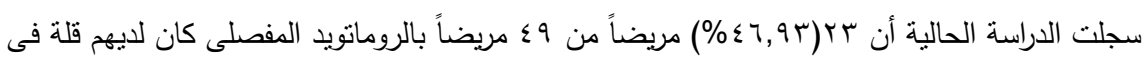

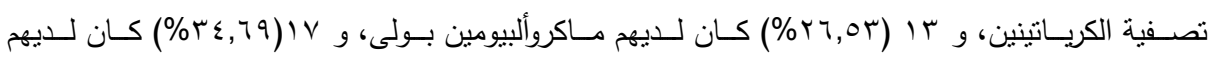

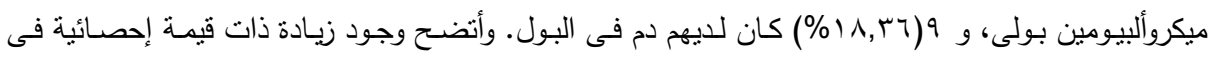

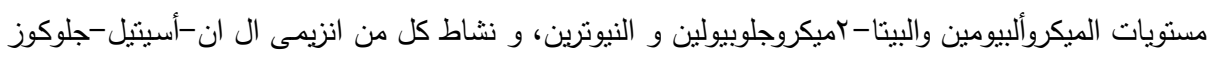

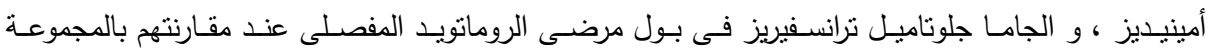

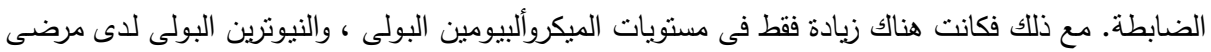

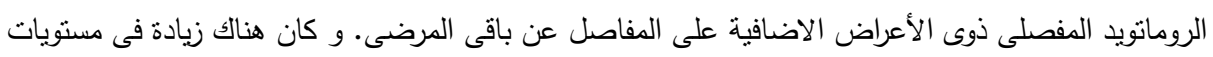

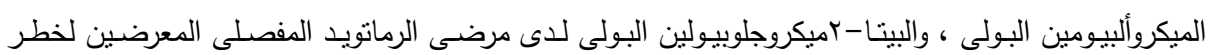

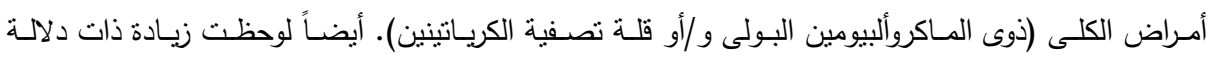

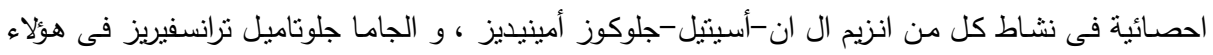

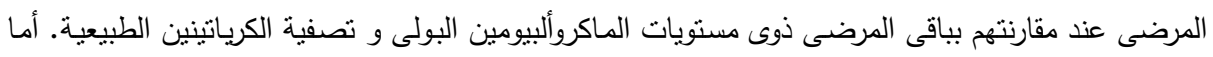

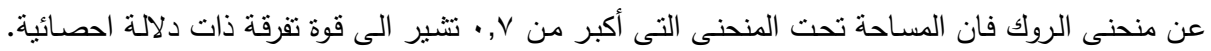

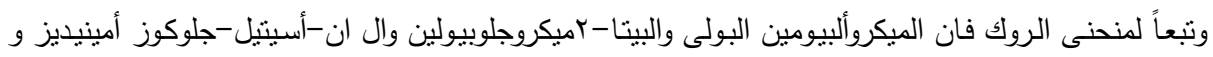


الجامـا جلوتاميل ترانسفيريز و النيوترين لهم قوة تفرقة متفاوتـة لمعرفة التأثر الكلوى بين مرضى الرومانويد

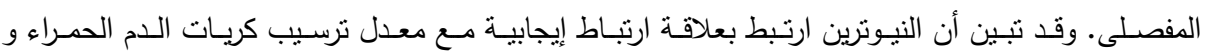
الميكروألبيومين البولى والبيتا - بميكروجلوبيولين. تثثير ننائج هذه الدراسة الى أن فحص البول المنتظم بطرق سهلة و حساسة للمعرفة المبكرة للتأثر الكلوى

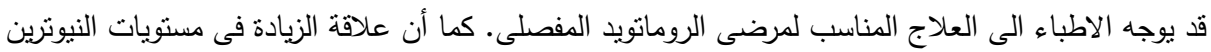

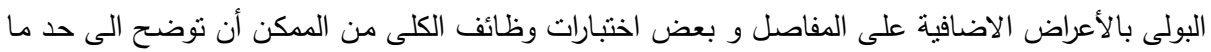

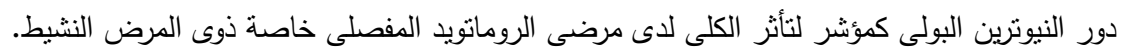

